\title{
NOVAM evaluation from aerosol and lidar measurements in a tropical marine environment
}

\author{
Gerrit de Leeuw and Gerard J. Kunz \\ TNO Physics and Electronics Laboratory \\ The Hague, The Netherlands
}

\begin{abstract}
NOVAM, the Naval Oceanic Vertical Aerosol Model, has been developed to predict the non-uniform and non-logarithmic extinction profiles that are often observed in the marine atmospheric boundary layer. The kernel of NOVAM is the Navy Aerosol Model (NAM) that calculates the aerosol size distribution at 10 in ASL from meteorological parameters. The aerosol profile is calculated from the surface layer size distribution with a physical model. Extinction profiles are calculated from the aerosol profiles using a Mie code. NOVAM requires validation in different meteorological scenarios. During the KEY90 experiment, July 1990 near Marathon (F1), NOVAM was validated in a tropical marine environment. We measured the surface layer particle size distribution profile at levels from 0.5 to 4 m ASL to evaluate the largeparticle end of NAM. The NOVAM prediction of the aerosol profile in the mixed layer was evaluated by lidar measurements of the $1.06 \mu \mathrm{m} \mathrm{backscatter}$ profile. The time-serial lidar measurements show the convective plumes and the variability in both the aerosol content at higher levels in the boundary layer and in the boundary layer height itself. Consequences for application of NOVAM for slant path transmission are discussed.
\end{abstract}

\section{INTRODUCTION}

For the assessment of electro-optical (EO) instrumentation for vertical and slant path observations, knowledge on the vertical variation of electro-magnetic scattering and absorption at wavelengths from the visible to the far infrared is important. Existing empirically derived expressions for the contribution of the aerosol to the Eo propagation characteristics in the marine atmosphere were formulated for single levels. An example is the Navy Aerosol Model $(\mathrm{NAM})^{1,2}$ as found in LOWTRAN VI. ${ }^{3}$ To extend the extinction prediction to higher levels, a physical model is required to calculate the vertical distribution of the aerosol. Empirical models fail to predict the correct behavior (see, e.g.,
ref. 4 for a brief review). Therefore the Naval Oceanic Vertical Aerosol Model (NovaM) has been developed. $2,5,6,7,8$ NOVAM uses meteorological profile information to account for the physical processes that influence the vertical aerosol structure and are thought to be responsible for the observed variety of profiles. NovaM has been designed to describe the non-uniform but also non-logarithmic aerosol distributions that are often observed throughout the marine atmospheric boundary layer (MABL).

NOVAM is a mixture of empirical and dynamical models. The kernel for NOVAM is NAM which has been extensively updated from the original. ${ }^{1}$ NAM produces an aerosol size distribution at $10 \mathrm{~m}$ above the sea surface, from input data of wind speed (both current wind speed and the 24-hour average), visibility and relative humidity. The NaM-generated surfacelayer particle size distribution is mixed throughout the MABL by turbulent-controlled processes, further modified by humidity effects. The physics describing these processes are determined by the MABL vertical structure. Various models describing the atmospheric vertical structure are included in NOVAM, such as a simple mixed-layer model ${ }^{9}, 10$ and a shallow convection case. ${ }^{10}$ Provision has been made to include other models such as for deep convection. The selection of the model is based on the input parameters describing the vertical stratification (thermal stability, the presence of an inversion and the inversion height), cloud cover, cloud type, wind speed, and the requested wavelength for the extinction calculation. An extensive description of NOVAM has been presented in refs. 2, 5 and 6 .

NOVAM will perform best when all of the required input parameters (see, e.g., ref. 2 or 5) are available. Thus the input files need to contain surface observations and the MABL vertical structure. The latter information can be obtained from a rawinsonde observation. If the information on the vertical structure is not available a default relative humidity profile, based on the surface observations, is generated. 11 This default profile is also used when the required input parameters do not satisfy the presently supported models (mixed-layer, shallow convection or stratus). The stratus 
mode $1^{12}$ is an empirical model that applies only to the marine stratus clouds for wind speeds less than $5 \mathrm{~m} / \mathrm{s}$ and a desired extinction calculation for wavelengths between 1 and $11 \mu \mathrm{m}$.

A prelininary NOVAM-estimated profile comparison with one set of experimental data yielded favorable results. ${ }^{6}$ A more comprehensive initial evaluation of NOVAM utilizing an extended aerosol and extinction data base obtained during the project FIRE (First ISCCP Regional Experiment) confirmed these. ${ }^{8}$

NAM is based on data collected over the world oceans. ${ }^{1}$ NAM has been evaluated by several users and has been updated from new experimental evidence. 1,13 The development of NOVAM was based on data collected over the Pacific near the Californian coast. The FIRE experiment took place in the same area, near San Nicolas Island. Therefore, the initial NOVAM evaluation from the FIRE data was basically a test for the performance of NOVAM in the same area where the data was taken in its initial development, but in different conditions. Further evaluation is required in different geographical areas with different meteorological and oceanic conditions. Although several data sets are available which might be used for evaluation purposes, they were not designed for this purpose and often one or more input parameters are missing. NOVAM performs best when all of these are available, and only in that case tests can be made on the influence of missing inputs. Therefore the KEY90 experiment has been organized as the next step in the NOVAM validation process, in a tropical/trade wind type of situation, where the physics are strongly different from those in the atmosphere over the Pacific.

\section{OBJECTIVES}

The general objective of the KEY90 experiment was to provide an environment in which enough quality measurement data could be obtained in order to verify the operation of NAM and NOVAM in a tropical ocean scenario. This was accomplished by simultaneously obtaining the meteorological parameters necessary to exercise NOVAM in its full capability and at the same time to provide a ground truth measurement of the extinction profile at various wavelengths to provide a standard by which to judge the extinction profile predictions provided by NOVAM based on the meteorological input. ${ }^{14}$ The objectives of the TNO Physics and Electronics Laboratory to participate in the KEY90 experiments were:

- to collect data for the determination of the source function for the marine aerosol in a tropical ocean scenario

- to use the same aerosol data for evaluation and improvement of NAM

- to determine the vertical structure of atmospheric backscatter and extinction coefficients, with a lidar system, for evaluation of NOVAM.

In this contribution we will only address the latter two objectives, the validation of NAM and NOVAM. For validation of NAM, surface layer aerosol data are required. Because the size distributions of the smaller particles were measured from the boat by another group (OMIST, Manchester, UR), we focussed on particles larger than $13 \mu \mathrm{m}$ in diameter which were measured with Rotorod impaction samplers. ${ }^{15,16}$ These samplers were employed from a boat, close to the optical particle counters at about 4 above mean sea level, and from a float to measure profiles. The concentrations of the large particles measured with the Rotorod samplers have the greatest influence on the extinction properties in the far IR. A comparison of NAM with the HEXMAX data set (North Sea, 1986) has shown a discrepancy at high winds. A reliable description of the influence of these large particles may require the addition of a fourth mode to NAM. 5,6

The other objective of this report is the evaluation of NOVAM using extinction and backscatter profiles measured with lidar. Lidar is a unique tool for remote sensing of atmospheric optical parameters which are of ten related to atmospheric boundary layer dynamical processes. Direct measurements of these processes usually requires airborne platforms which are expensive to operate, but have the advantage that they can cover large areas in a limited time. Lidar can be operated semi-continuously from a fixed point, yielding time series of boundary layer profiles which show its evolution over the range of the lidar. Lidar systems can also be used from airborne platforms which have the same disadvantages as other airborne measurements (high cost and limited operation time), but can measure the spatial variability of the boundary-layer vertical structure.

The information obtained with lidar can be used to better understand atmospheric processes and to test boundary layer models. NOVAM is based on such models. Therefore, lidar directly yields the information required as 'ground truth' for validation of NOVAM profiles calculated from meteorological parameters. An additional advantage is that extended time 
series can be measured with lidar. During KEY90 we measured up to 14 hours, but also unattended operation during days is feasible when safety can be guaranteed. ${ }^{17}$ Such time series are useful to test the forecasting capability of NOVAM which has to be developed yet. NOVAM is a candidate for forecasting because it is based on physical equations (cf. ref. 18).

\section{THE KEY90 EXPERIMENT}

The KEY90 experiment took place from July 2 to July 19, 1990, in the Straits of Florida in the area centred around Marathon (Florida, USA). The location, in combination with boat and aircraft measurements, offers the opportunity to be away from land influences and major continental effects on the data. KEY90 was a cooperation between institutes from the USA (Naval Research Laboratory, Washington D.C.; Naval Ocean Systems Center, San Diego, CA; Naval Postgraduate School, Monterey, CA), the UR (UMIST, Manchester) and The Netherlands (TNO Physics and Electronics Laboratory, The Hague). A general overview of the KEY90 experiments has been presented in refs. 19 and 20 . A brief summary is presented below.

The experiments were centred around Marathon, FL, USA (about $80.06 \mathrm{~W} ; 24.40 \mathrm{~N}$ ), the home base of a small boat that was used for surface measurements of aerosols and meteorological parameters, as well as for radiosonde launches. other surface data were collected ashore in Marathon. Data on the vertical structure of extinction and backscatter, from aerosol and lidar measurements, and temperature and humidity profiles which are required to calculate the extinction profiles with NOVAM, were collected with aircraft, ground-based lidar equipment and radiosondes. The aircraft included the NRL P3 which flew the aureole lidar ${ }^{21}$ and the NOSC airborne platform that was also used in other NOVAM evaluation experiments. ${ }^{8}$ Radiosondes were launched by NPS, both from the boat and ashore in Marathon. other meteo parameters were collected at Marathon airport. Visual weather observations were made by various participants.

The TNO Physics and Electronics Laboratory participated in these experiments with Rotorod equipment and a small lidar system. Rotorod rotating impaction samplers (cf. ref. 15) were used from a boat at some tens of miles from the coast to measure aerosol size distributions. Profiles close to the water surface $10.5 \mathrm{~m}$ to $1.25 \mathrm{~m}$ above the instantaneous air-sea interface) were obtained with the Rotorods mounted on a float which drifted about $15 \mathrm{~m}$ upwind from the boat. In addition, particle size distributions were measured aboard the boat at about 4 above the mean sea surface, close to the UMIST optical particle counters. These aerosol sampling methods yield complementary information. The vertical structure of the backscatter at a wavelength of $1.06 \mu$ mas measured with the TNO Mini lidar system (wavelength $1.06 \mu \mathrm{m} ;$ see, e.g., ref. 22 or 19 for details), to an altitude of about $2 \mathrm{~km}$. To this end, the lidar was used under an elevation angle of $60^{\circ}$. The profiles were measured with a frequency of 2 per minute. Information on cloud reflections at higher altitudes was also obtained. The THO lidar equipment was mounted ashore in Marathon, on the eighth floor of an apartment building at about 150 from the beach. 19

Detailed descriptions of our experiments and a survey of the data collected during KEY90 can be found in ref. 19. A survey of the whole experiment, including a detailed case study, is presented in ref. 20.

\section{NAM EVALUATION FROM AEROSOL MEASUREMENTS (D>10 $\mu \mathrm{m})$}

In the initial presentation of NONAM, ${ }^{4}$ the model has been critically evaluated. As part of this evaluation the formulation of NAM for the largest particles has been discussed. The concentrations of these particles, described as the 'fresh' marine mode in NAM, are given by a log-normal distribution with a mean radius of $r_{3}=2 \mu \mathrm{m}$, a fixed width, and an amplitude which is only determined by the current wind speed and the relative humidity:

$$
\begin{aligned}
& n(r)=A_{3} \exp \left(-\ln \left[\frac{r}{f_{3} r_{3}}\right]^{2}\right), \\
& r_{3}=2.0 \mu \mathrm{m} \quad A_{3}=\operatorname{MAX}\left(1.510^{-5}, 0.01527\{u-2.2\}\right)
\end{aligned}
$$


This formulation is based ${ }^{2}, 13$ on aerosol particle size distributions measured in the North atlantic ${ }^{15}$ with the same impaction method applied during KEY90. Nevertheless, the comparison in Fig. 1a shows that the concentrations of the particles measured during the KEY90 experiments are on average about one order of magnitude higher than predicted by NAM from the simultaneously measured meteorological data. One obvious reason for this discrepancy could be the height at which the particle size distributions were measured. However, the concentration gradients between the 10 in level, for which NAM was developed, and the average level of 4 AMSL, where the present data were collected, are usually not large at the wind speeds occurring during the KEY90 experiments $(2-8.5 \mathrm{~m} / \mathrm{s})$. Similar comparisons as in Figure 1 for the other levels at which samples were taken during KEY90 show the same trend. Although the concentrations are certainly not constant with height, they are only occasionally sinilar to the NaM-predicted values. In all other cases they are significantly higher.

A more honest evaluation of NAM would be the use of data taken at approximately 10 aSL. Since these are not available from KEY90, we have used data collected during the BEXOS experiments in the Fall of 1986 in the North Sea ${ }^{16}$ to check on the height dependence between 4 and $10 \mathrm{~m}$. Here too, some height dependence has definitely' been observed but the differences are not large and the concentrations are within the same upper and lower limits. The comparison between the HEXMAX data for 3-5 and the particle size distributions predicted by NAM from simultaneously measured meteorological data is shown in Figure $1 b$.

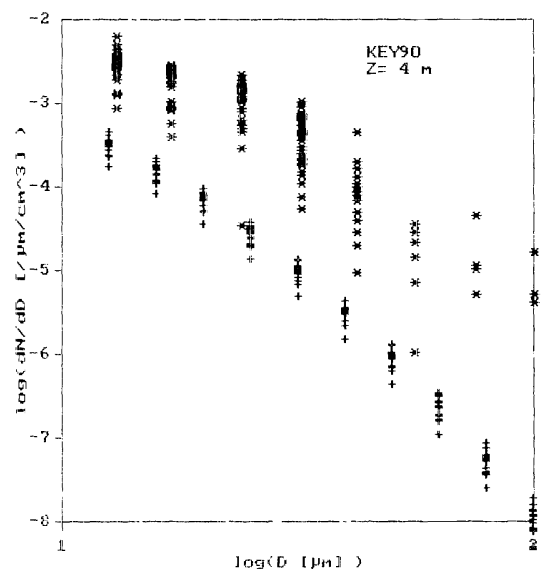

(a)

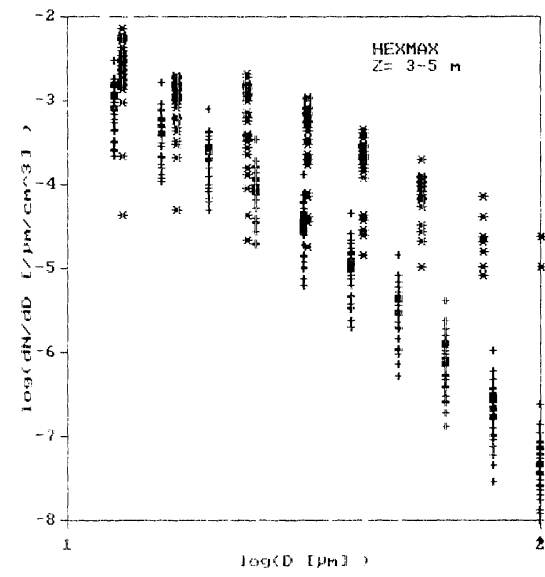

(b)

Figure 1.

a) Comparison between particle size distributions measured during KEY90 from the boat at an average level of 4 in above mean sea level $(*)$, and particle size distributions predicted by NAM from simultaneously measured meteorological data

b) same for HEXMAX data measured at levels between 3 and $5 \mathrm{~m}$ AMSL from a tower in the North Sea. 16

Comparison between Figures $1 \mathrm{a}$ and $1 \mathrm{~b}$ shows some salient features which bear importance for the large particle mode of NAM:

- the concentrations of the KEY90 and the HEXMAX data are quite similar: apart from some outlyers both the upper and the lower limits are about the same

- the NAM-predicted concentrations for KEY90 vary over a wuch swaller range than the experimental data, while for HEXMAX these ranges are similar

- the discrepancies between NAM and the HEXMAX data is on average smaller than between NAM and the KEY90 data; this is mainly due to the larger range of wind conditions during HEXMAX, resulting in larger variations of the NAM-predicted concentrations

- the discrepancies between NAM and the measured concentrations increase as particle size increases, or in other words, NAM predicts too large a slope for the particle size distributions. This applies to both data sets.

- however, the KEY90 concentrations of the largest particles (D>56 $\mu \mathrm{m}$ ) are significantly smaller than those for the HEXHAX data 
Because the NAM large particle mode is based on observations in the North Atlantic, ${ }^{15}$ this is in fact a comparison between three data sets, collected at different geographical locations and in different meteorological and oceanographic conditions. Some of these have been collected in table 1.

Table 1. Conditions for the three data sets used in the NAM evaluation

\begin{tabular}{|c|c|c|c|c|}
\hline data set & location & $\begin{array}{l}\text { wind speed } \\
{[\mathrm{m} / \mathrm{s}]}\end{array}$ & watef temp. & $\begin{array}{l}\text { wave and } \\
\text { meteo condition }\end{array}$ \\
\hline $\begin{array}{l}\text { Cumulus } \\
\text { June } 1983\end{array}$ & North Atlantic & $2-13$ & $10-11$ & $\begin{array}{l}\text { open ocean: } \\
\text { swell and wind waves }\end{array}$ \\
\hline $\begin{array}{l}\text { HEXMAX } \\
\text { Fall } 1986\end{array}$ & North Sea & $2-24$ & $10-14.5$ & $\begin{array}{l}\text { mainly wind waves, tidal currents } \\
\text { variable conditions, many fronts causing confused seas }\end{array}$ \\
\hline $\begin{array}{l}\text { KEY90 } \\
\text { July } 1990\end{array}$ & Straits of Florida & $2-8.5$ & $29-30$ & $\begin{array}{l}\text { variable wind speed resulting in unbalanced wave field } \\
\text { low winds, convective situation }\end{array}$ \\
\hline
\end{tabular}

The most striking observations are the similarities between the concentrations measured during KEY90 and BEXMAX, while the NAM-predicted ranges of concentrations for KEY90 are so much different from the experimental data. The small range of concentrations predicted by NAM for KEY9O is a consequence of both the small range of the wind speeds and their low values during this experiment. The question is why the range of observed concentrations was so much higher and even similar to the range of concentrations observed in wind speeds of up to $25 \mathrm{~m} / \mathrm{s}$ during the HEXMAX experiments? The onset of whitecapping depends on meteorological conditions and has been observed between wind speeds of 4 and $7 \mathrm{~m} / \mathrm{s}$. The whitecapping ratio ${ }^{23}$ varies approximately with wind speed as $\mathrm{u}^{3.5}$. Accordingly, at the low wind speeds occurring during KEY90 whitecapping should usually have been quite insignificant and consequently the production of sea spray aerosol should have been small. The analysis of the aerosol concentrations measured during KEY90 shows that they are indeed fairly independent of wind speed: although the lowest concentrations were observed at about $2.5 \mathrm{~m} / \mathrm{s}$, for higher wind speeds there is no clear trend showing a definite wind speed dependence. This conclusion is not supported by the analysis of the other two data sets. Although in both the North Atlantic and the HEXMAX data sets the data for wind speeds smaller than $8 \mathrm{~m} / \mathrm{s}$ are too sparse for statistically sound analysis, the trends in these data is to increase with wind speed (cf. Figure 5 in ref. 15). Apparently some other mechanism obscures the wind speed dependence in the KEY 90 data set. Candidates are the wave development stage and efficient transport due to convective mixing. Young wind waves, which are not in balance with the wind field, are steeper and break more frequently than aged waves. Hence, as the wind picks up, initially more breaking occurs than in steady state conditions. In terms of aerosol physics, this implies that more aerosol is produced than according to a model based on the current wind speed alone, irrespective of recent wind history. Relatively fast increases in wind speed did occur during KEY90, and since the wind speed was often around the value where the onset of whitecapping occurs, young waves may frequently have been generated, resulting in more wave breaking than in accordance with a prediction based on mean wind. In NAM, wind history (24-hours mean wind speed) is only used to predict the concentrations of the 'aged' marine mode (mode radius $0.24 \mu \mathrm{m}$ ).

The second mechanism which should be considered is aerosol transport due to convective mixing. The lidar measurements (see section 5) show that often a convective situation occurred during KEY90, with aerosol-containing eddies rising up, alternated by sinking cells of clear air. Obviously such mixing processes affect the aerosol concentrations. Sea spray aerosol produced at the surface will be lifted in rising eddies and thus be easier transported upward than in neutral situations. This gives rise to greater concentrations away from the surface. On the other hand, the concentrations will be smaller due to dilution by clean air. The concentrations which are measured in such cases depend of course on the sampling time and the size of the eddies, as well as the type of eddy, rising or descending, that is being sampled.

Another mechanism which should be considered to explain the variability of the aerosol concentrations during KEY90 is sea spray production by rain. ${ }^{24}$ Intensive showers often occurred during KEY90, and although care was taken that the measurements were made outside of these, rain-produced aerosol could have been advected over a relatively short distance to the sampling position. Thus even for the very large particles considered in this study, advection of particles produced elsewhere should be considered. 
The high aerosol concentrations as compared to those measured both in the North Atlantic and in the North Sea is another important question. As indicated above, several mechanisms may be responsible for the stronger variability of the aerosol concentrations than expected from the current wind speed alone, such as the creation of a non-steady state situation with more wave breaking, convective mixing and production due to rain. On the other hand rain may also be responsible for the removal of aerosol by wet deposition while the concentrations are also diluted by convective mixing with clean air. Therefore, another mechanism should be considered that could be responsible for the high concentrations. As indicated in ref. 25, the aerosol production rate is higher in warm water than in cooler water. As the water temperature goes up the volume of air entrained goes into more and more smaller bubbles than in cold water. In addition, the number of jet drops produced per bubble decreases as the size of the bubble increases. Consequently, in warm water more jet drops are produced than in cold water both because more bubbles are formed during each wave breaking event and because more droplets are produced from each bubble. Since the water temperature during KEY 90 was around $29^{\circ} \mathrm{C}$ and during HEXMAX only $10-12^{\circ} \mathrm{C}$, this could explain the similar concentrations in spite of the much higher wind speeds during HEXMAX. The lower concentrations observed in the North Atlantic, as compared to those observed during BEXMAX, are likely due to the longer waves in the open ocean.

The variability of the aerosol concentrations during KEY90 has further been demonstrated from the case study for 14 July. ${ }^{20}$ The wind speed during the measurements on 14 July was fairly constant between 7 and $8 \mathrm{~m} / \mathrm{s}$, relative humidity varied between $76 \%$ and $83 \%$, but the particle concentrations varied between about a factor 2 and more than a factor 3 (depending on particle size), see Figure 2. The latter variations cannot be understood from the wind speed and humidity changes alone. Sampling strategy has been considered (a measurement at $4 \mathrm{~m}$ above sea level from a rocking and rolling boat is likely to sample over a height range of several meters resulting in a particle size distribution which is an average over these heights) and mixing processes. The profiles measured during this case show that at the beginning of the day significant gradients occurred between 4 and below $1.25 \mathrm{~m}$, which disappeared in the course of the experiments. Unfortunately no data are available from levels between $4 \mathrm{~m}$ and $1.25 \mathrm{~m}$. In the absence of a gradient, the measured average particle size distribution is still representative for the mean height, but when a strong gradient is present this no longer applies.
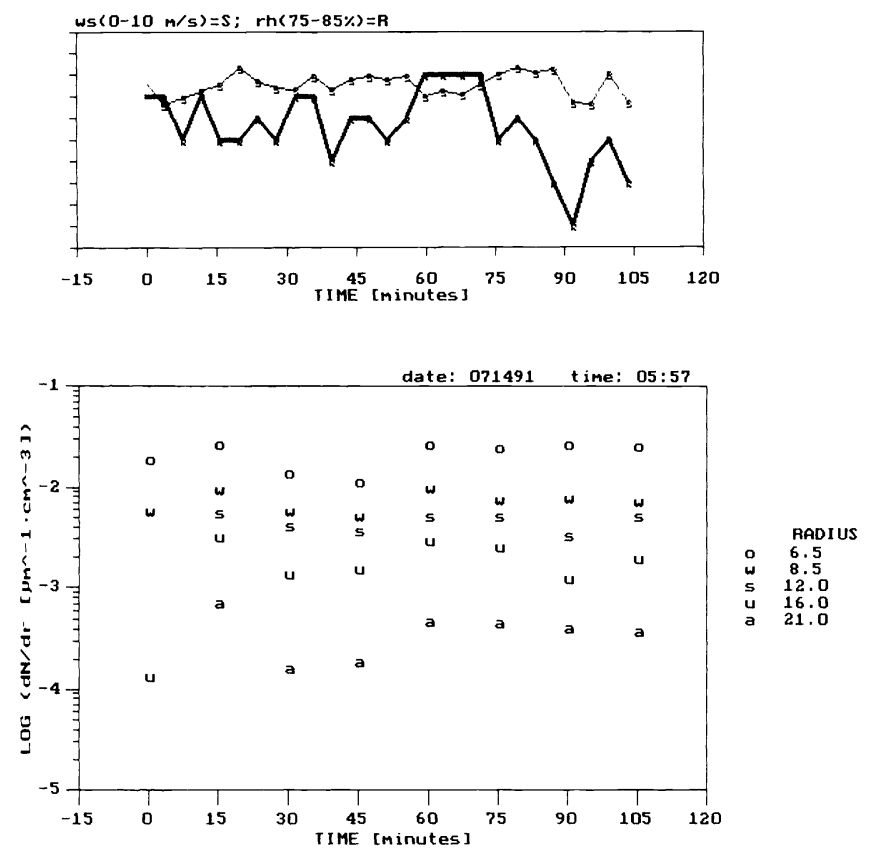

Figure 2. Temporal variations of particle concentration $\mathrm{dN} / \mathrm{dr}$, relative humidity and wind speed, on 14 July 1990. Starting time is 5:57 a.m. lower panel: $\mathrm{dN} / \mathrm{dr}$ for particles of 6.5 (0), 8.5 (w), 12 (s) $16(\mathrm{u})$ and 21 (a) $\mu \mathrm{m}$ in radius, measured with Rotorod samplers from the deck of the boat at an average height of 4 in AHSL.

upper panel: relative humidity (bold line) and wind speed thin line). Note that the relative humidity has been plotted on a scale from $75 \%$ to $85 \%$, and the wind speed on a scale from $0 \mathrm{~m} / \mathrm{s}$ to $10 \mathrm{~m} / \mathrm{s}$.

The final point we discuss here concerns the slope of the particle size distribution. Both the KEY90 and the HEXMAX data show that these are smaller than predicted by NAM. As discussed before, the NAM predictions are for a lognormal size distribution with mean radius of $2 \mu \mathrm{m}$ and fixed width. Apparently the width of the particle size distribution is not 
correct for sizes between 10 and $100 \mu \mathrm{m}$. Apart from the production process, the concentrations of these particles are determined by the balance between turbulent transport (and convective mixing in this situation) and gravitational fallout. Particles stay in suspension when $\mathrm{W} / \mathrm{ku}_{*}<1,26$ where $\mathrm{W}$ is the gravitational fallout velocity, $\mathrm{k}$ is the Von Karman constant $(0.4)$ and $u_{*}$ is the friction velocity. As wind speed increases more particles stay in suspension and hence the spectrum shifts toward larger sizes. Figure 1 shows that the discrepancy between NAM and the experimental data increases with particle size, which leads to the conclusion that particle mixing is apparently insufficiently taken into account by NAM. Investigations are being made into the variation of the spectral slope of the particle size distributions as function of meteorological parameters and as function of height, for the data sets discussed above, which should lead to improvement of the parameterization of the concentrations of the largest particles.

\section{LIDAR OBSERVATIONS AND IMPLICATIONS FOR NOVAM}

NOVAM requires information on the structure of the marine atmospheric boundary layer for optimum performance. Usually this information will be available from a rawinsonde sounding (during KEY90 this information was also obtained from aircraft measurements). Rawinsonde soundings provide a point measurement (in time) of the profiles of air temperature and dew point (or absolute humidity), from which the relative humidity profile, cloud level, and the inversion height are determined for use in NovaM. These parameters are usually not constant in time and appreciable variations may occur. Rawinsonde soundings are made only a few times per day and several questions have come up regarding the validity of such a sounding as regards the prediction of the slant path transmission of visible/IR radiation. In particular in a convective situation as encountered in the KEY90 tropical marine environment this may be of importance because convective cells containing high aerosol concentrations are alternated by sinking eddies with clean air that has been mixed in from the free troposphere above. Remote sensing with lidar from a ground-based station is a suitable technique to investigate these processes because it can be operated continuously over extended periods of time to measure the boundary layer structure from close to the surface to above the top of the boundary layer.

Based on our lidar measurements, we have investigated the following questions:

- what is the value of a NOVAM prediction of the slant path transmission based on a point measurement (in time and space) of the atmospheric structure from a rawinsonde sounding?

- given a rawinsonde sounding of the vertical structure of the MABL, what is the variability in the slant path transmission during the next hours, i.e. what is the error in the slant path transmission when the atmospheric structure information is not up to date?

- $\quad$ can lidar be used to predict the standard deviation in the NovaM prediction?

Obviously the answers to these questions strongly depend on the meteorological situation. Therefore the discussion below specifically applies to the KEY90 scenario. However, lidar has not been used in earlier NOVAM evaluations and the answers it can provide are important for the assessment of NOVAM. In addition, lidar data are available from several other experiments in the marine atmosphere ${ }^{4,27}$ which can be used for a similar analysis as presented below.

In addition to these questions, lidar can of course also be used for a direct comparison with NOVAM. This was the main reason for bringing the TNO lidar system to KEY90. However, only a few rawinsondes were launched near the lidar site in Marathon (Fl.) and all others were made at 2-3 hours sailing downwind.

Throughout this paper, lidar results will be presented as backscatter data because this is the parameter that is directly measured. Transmission losses were ignored because the visibility was always high (except in rain showers). This could have caused a maximum error of $18 \%$ at the far end $(2 \mathrm{~km})$. Extinction can be derived from the lidar data by solving the lidar waveforms, but this would have introduced large errors in these clear-weather situations.

\subsection{VARIABILITY OF THE BOUNDARY LAYER STRUCTURE}

The variation in time of the two-minutes averaged backscatter coefficients on $14 \mathrm{July}$, at an altitude of $80 \mathrm{~m}$, is shown in Figure 3 together with air temperature and relative humidity. The rise in the backscatter coincides with the temperature increase, while the strong decrease in the backscatter after 9:30 occurs almost simultaneously with the period when the relative humidity drops. The decrease in backscatter could be caused by the evaporation of aerosol particles in response to the decreasing humidity. The rise in the backscatter between 8:00 and 9:30, simultaneously with the increase in temperature, is not readily understood. At the same time the wind picked up, although whitecaps were not 
observed untii later that day. Nevertheless, the increasing wind may have caused some additional aerosol production and lifting by turbulent transport, resulting in increasing backscatter.
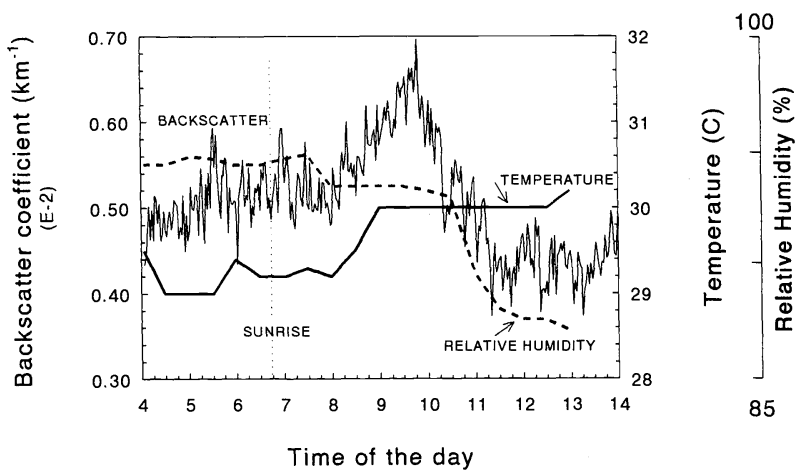

Figure 3. Backscatter coefficients in $\mathrm{km}^{-1}$ at an altitude of 80 . laveraged over 2minute intervals), air temperature and relative humidity, from 04:00 an to 02:00 $\mathrm{pm}$ on 14 July.

The convective situation is well illustrated by the false-color plots of the backscatter profiles in Figure 4 , for the day segment from 04:02 an to 06:27 an on 14 July 1990. Backscatter has been color-coded in these altitude versus time plots. Unfortunately, in this $\mathrm{b} / \mathrm{w}$ reproduction the backscatter scale is hard to distinguish, but the convective plumes are still visible. Note that in this presentation each profile has been repeated twice. The convective plumes are observed throughout the day. This is confirmed by the results from the aureole lidar flown in the NRL P3 aircraft [Hooper, private communication].

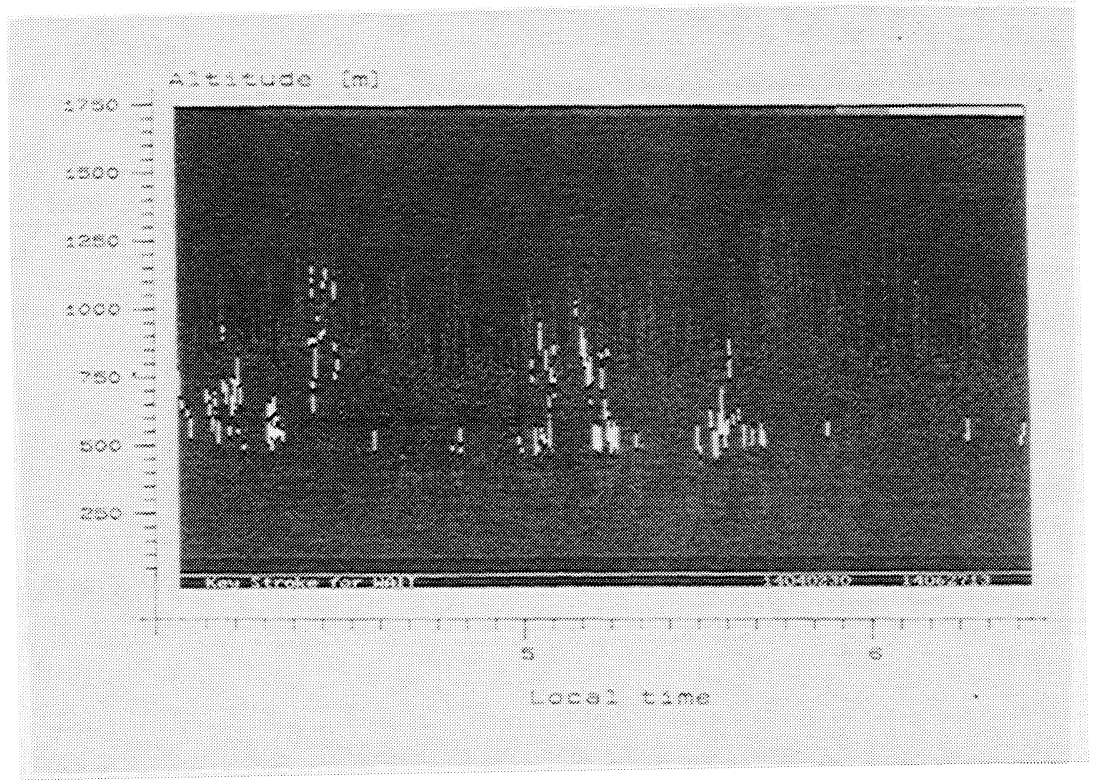

Figure 4. Backscatter profiles measured on 14 July 04:02-06:27 an, coded in false color. The color scale ranges from black to white (in the rhs top of the Figure) and runs from $\log (0.001 / \mathrm{km})$ to $\log (0.1 / \mathrm{km})$.

Cloud patches are observed as bright spots (high backscatter). They are more frequently observed before sunrise. The height of the mixed layer varies around $450 \mathrm{~m}$, which is confirmed by the results from the airborne lidar and by the rawinsonde. Diurnal effects are not observed. The backscatter below the clouds increases gradually. Also between the clouds the backscatter was often observed to increase. In these cases the increase in relative humidity caused condensation on the aerosol particles resulting in particle growth and higher backscatter, but apparently the humidity was too low to cause cloud formation.

Variations in the top of the boundary layer, viz. the mixed-layer height and the top of the entrainment layer, were determined from lidar profiles averaged over 10 minutes and their standard deviation. The profiles of the standard deviations provide information on the degree of mixing versus altitude. ${ }^{28}$ Small standard deviations have been observed at low altitudes, where the atmosphere is well mixed, and above the boundary layer. From altitudes of about 100 in to the 
top of the mixed layer the standard deviations increase with altitude due to the mixing of clear air from above. The standard deviations reach a maximum in the entrainment layer. This is illustrated in Figure 5.

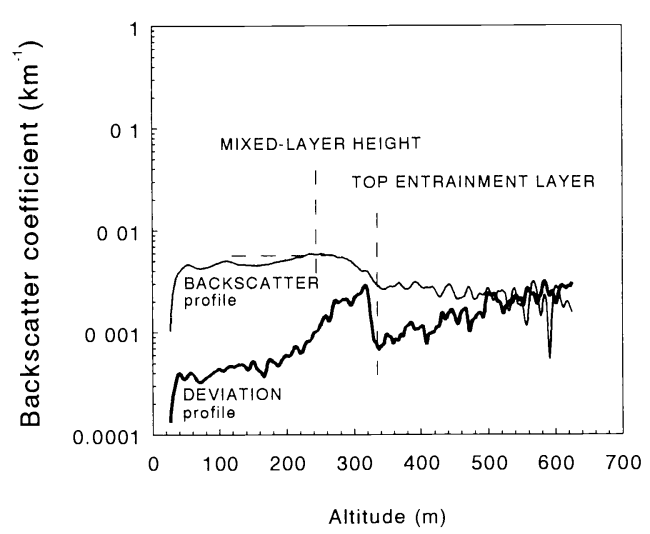

(a)

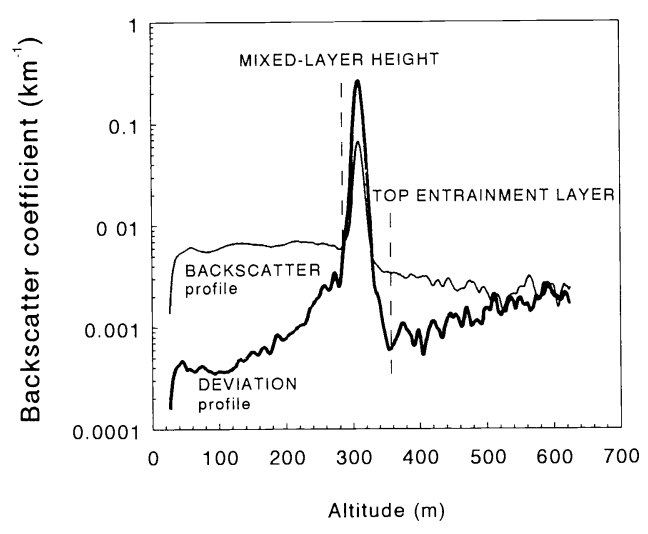

(b)

Figure 5. Examples of profiles of the 10-minute averaged backscatter coefficients (thin line) and standard deviations (bold line) in clear sky (a) and in clouds (b). The mixed-layer height and the top of the entrainment layer are indicated.

The mixed-layer height and the top of the entrainment layer have been determined from 10-minute averaged backscatter profiles and their standard deviations according to the following procedures.

1 . in the case of a clear atmosphere the mixed-layer height was defined as that height where the gradient in the average backscatter is zero, just before it decreases. The top of the entrainment layer is determined at the position of minimum backscatter combined with the minimum in the standard-deviation profile, after the sharp last ninimum (cf. Figure 5a). 2 . in the case of clouds the mixed layer height was defined at the position where the average backscatter starts to increase just before the cloud reflection (actually this is the cloud condensation level). The top of the entrainment layer was defined at the first minimum in the standard-deviation profile after the last cloud reflection (cf. Figure $5 b)$.

Results from application of this procedure are shown in Figure 6. The mixed-layer height varies between about 330 m and $550 \mathrm{~m}$. The top of the entrainment layer was observed at levels between 50 and 300 above the mixed-layer height (except for the cloud reflections before sunrise). The radiosonde launched in Marathon at 09:47 indicates a first inversion at $430 \mathrm{~m}$ and a second one at $715 \mathrm{~m}$. These values compare favorably with those derived from the lidar data. The rawinsonde sounding at about 07:00 am was made at some hours sailing upwind from Marathon. Therefore it is not surprising that these data are different from the lidar-derived values.

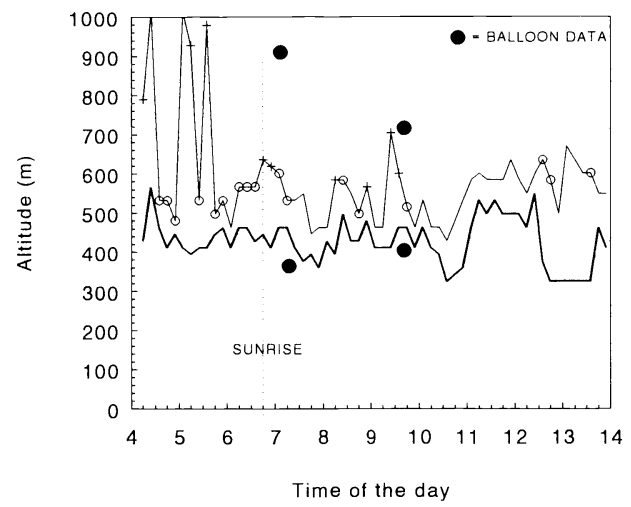

Figure 6. Mixed-layer height (bold line) and top of the entrainment layer (thin line) on 14 July from 04:00 am to 02:00 pm, as derived from backscatter profiles with the wo mini lidar in Marathon, Circles indicate the occurrence of one cloud reflection, plus signs indicate more than one cloud reflection in the 10-minute interval. Inversions obtained from the rawinsonde soundings are indicated by filled circles. 


\subsection{IMPLICATIONS FOR THE SLANT PATH TRANSMISSION DERIVED FROM A POINT MEASUREMENT}

As indicated above, we have chosen to measure only backscatter with the lidar to avoid problems associated with the solution of the lidar equation in a clear atmosphere. ${ }^{29}$ In contrast, the NRL aureole lidar measured extinction profiles [Hooper, private communication]. Comparison of these extinction values with our backscatter coefficients shows that the extinction/backscatter ratios were about 10, in good agreement with earlier observations. Hence our backscatter profiles could be easily transformed to extinction profiles by multiplication with a constant. However, for the analyses presented in this paper this would not yield any additional information. Therefore we have chosen to use the backscatter also for our investigations of the effect of a point measurement on the value of the slant path transmission $\mathrm{T}$ :

$$
T(R)=\exp \left\{\int_{0}^{R}-C B(r) d r\right\}
$$

where $B(r)$ is the range-dependent backscatter coefficient.

To investigate the influence of the mixed-layer height variations on the slant path transmission the integral of the exponent in eq. 3 has been solved and the relative difference with respect to the initial value at an arbitrary chosen starting time has been calculated for the for the remainder of the measurements of that day. The relative difference was calculated as:

$$
\epsilon=\frac{p i b(\text { tref })-p i b(t)}{\operatorname{pib}(\text { tref })}
$$

where $\mathrm{pib}($ tref) and pib(t) are the path-integrated backscatter at the arbitrary chosen reference time and at some time $t$ there after, respectively. Integration limits for the calculation of pib were from 25 m (the altitude at which the lidar was mounted in an apartment building in marathon) to $400 \mathrm{~m}, 500 \mathrm{~m}$, or $600 \mathrm{~m}$. These levels were chosen as the heights at which the top of the boundary layer was observed (see Figure 6). In the following discussion $\epsilon$ is considered as the error introduced by not using an instantaneous sounding for the boundary layer structure.

Results presented in Figure 7 are for 14 July. The calculations show that the largest errors occur at the top of the boundary layer. Although errors as large as $50 \%$ were occasionally observed in the pib between 25 and $400 \mathrm{~m}$, the average was about $10 \%$ (Figure 7a). However, when the integrations are performed up to $600 \mathrm{~m}$, the pib is $50 \%$ or more smaller, except when clouds are in the lidar path. This means that the pib based on the reference profile would be a factor 2 too high for non-cloud situations! The error increases very fast after the reference time at 4:00 am. Apparently this happens to be a case where the 'initial sounding' was made just before a cloud, thus indicating atypical conditions. When the same sounding is also used for the following five hours the errors will obviously remain high. A new sounding improves the situation (Figure 7c), but in that case too, errors of up to $30 \%$ are already observed in the first hour. After $10 \mathrm{am}$, the backscatter drops as was already shown in Figure 3. This results also in a decrease of the pib, thus giving rise to errors varying between about $10 \%$ and $50 \%$ if the meteorological inputs are not updated. It must be noted here that the pib is not solely determined by the sounding but also by the surface observations which can be made more frequently. However, the variations displayed in Figure 7c are unlikely to be covered by updating the surface observations alone (see e.g. Figure 3 for temperature and humidity values; wind speed varied only little). Apart from the backscatter values, also the boundary layer height dropped appreciably around 10:00 am. 

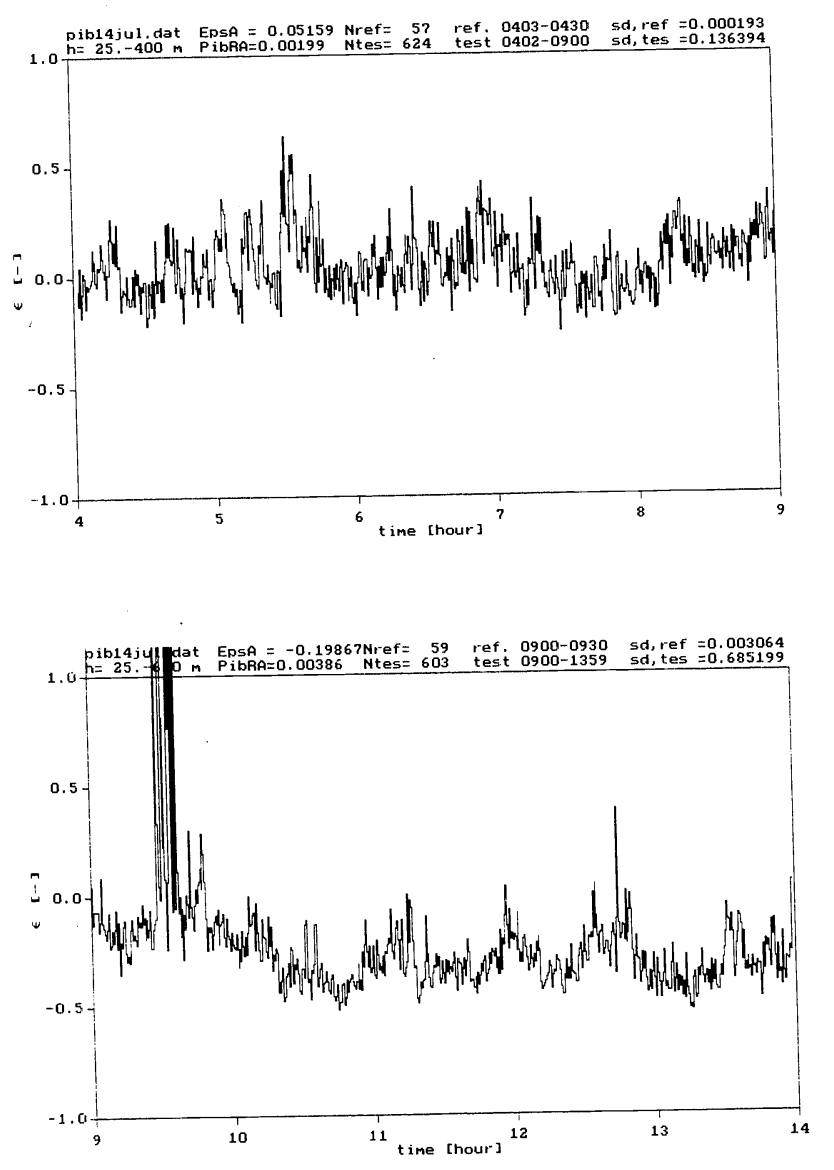

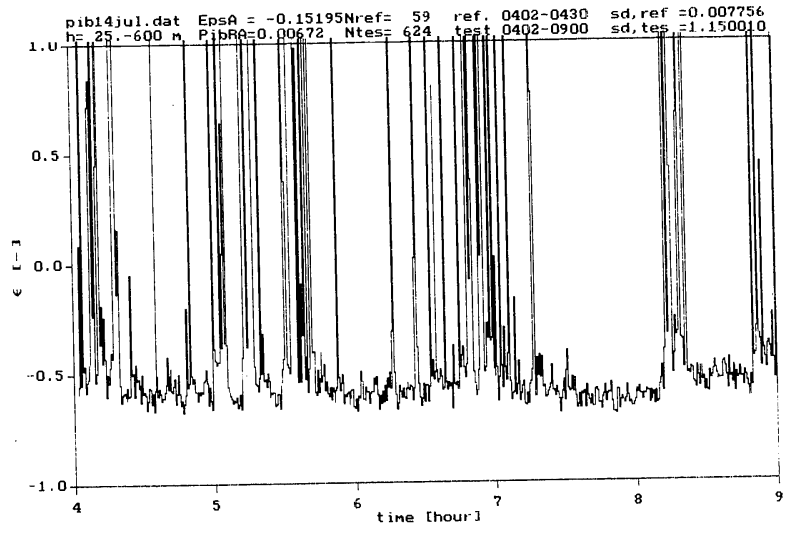

Figure 7. Relative differences $\epsilon$ (eq. 4 ) in the predicted slant path transmission

as function of time.

a) the reference path-integrated backscatter pib(tref) has been taken as the average from 4:00 to 4:30 am, the integration was performed from $z=25$ in to $z=400$. b) same as Figure a, but integrated from $z=25$ to $z=600$ in

c) same as Figure $b$, but the reference has been taken as the average pib between $9: 00$ and $9: 30$ am.

Note that the spikes are caused by the large pathintegrated backscatter caused by clouds.

\section{DISCUSSION}

Aerosol and lidar measurements have been used to validate the 'fresh marine' mode 3 of NAM (mode radius $2 \mu \mathrm{m}$ ) and the validity of a slant path transmission derived from a point measurement as used by NOVAM to calculate the extinction profile. Large discrepancies were observed between the measured aerosol particle size distributions and those predicted by NaM from simultaneously measured meteorological parameters. Comparison of these results with those based on data obtained in experiments in the North Sea and in the North Atlantic indicates that the parameterization of NAM based only on wind speed and relative humidity is unsatisfactory. Other parameters should be taken into account. obvious candidates are the water temperature, the recent wind history, and convective and turbulent mixing processes.

The water temperature affects the spectrum of the bubbles from which the sea spray aerosol is produced. ${ }^{25}$ As water temperature rises more bubbles are produced from an air volume and the bubble spectrum shifts to smaller sizes than in colder water. This effect is enforced because smaller bubbles produce more droplets per bubble. Experimental evidence for this effect has not been produced from field experiments thus far, and the above considerations are based on laboratory experiments in different tanks and different conditions. ${ }^{25}$ However, the occurrence of such an effect could explain why the concentrations observed during KEY 90 in the tropical ocean $\left(t_{\text {sea }} \approx 29.5^{\circ} \mathrm{C}\right)$ in relatively low wind speeds $(2-8.5 \mathrm{~m} / \mathrm{s})$ match so closely the range of concentrations observed during the HEXMAX experiments in the North Sea $\left(t_{\text {sea }} \approx 12^{\circ} \mathrm{C}\right)$ in wind conditions which were most of the time over $10 \mathrm{~m} / \mathrm{s}$, with maxima of $24 \mathrm{~m} / \mathrm{s}$ !

The recent wind history determines wave age. Young waves are steep and break wore frequently than those in balance with the wind field. Hence more aerosol is produced when wind speed increases. This effect might be taken into account by using the friction velocity $u_{*}$ instead of the wind speed. Higher values for the friction velocities have been observed 
in both increasing and decreasing wind speeds. ${ }^{30}$ The effect of wave age on $u_{*}$ has recently been discussed by, e.g., Blake $e^{31}$ or Maat et al. ${ }^{32}$

Turbulent mixing is usually also determined by the wind speed or friction velocity, except when strong thermal stratification occurs. Due to thermal mixing, more particles are lifted from the production zone and larger particles stay in suspension. This affects both the concentrations and the shape of the particle size distribution.

In the situation encountered in the tropical ocean during KEY90, convective mixing appeared to be important. Both the airborne aureole lidar observations and our ground-based measurements clearly show the convective plumes with aerosol alternated by clean air cells.

These two mixing effects, together with a variable production rate, in a situation with relatively low wind speeds which does not keep the particles in suspension, could explain the observed variability in both the aerosol concentrations and in the lidar backscatter (see Figures 1 and 3). This variability is both much larger than observed in similar wind speeds during other experiments where the same technique was used, and much larger than predicted by NAM.

The results presented here are not conclusive in the sense that they can be incorporated in NAM. The aerosol data will be used together with other data bases to further investigate the effects mentioned above, including the spectral shape as function of height and as function of meteorological parameters and the effect of water temperature on the concentration. For this purpose data are now available for water temperatures from $0^{\circ} \mathrm{C}$ to $30^{\circ} \mathrm{C}$, wind speeds up to 25 $\mathrm{m} / \mathrm{s}$, and a range of thermal stabilities including the convective situation during KEY 90 . This may lead to a better parameterization of NAM for the largest particles and/or the inclusion of a fourth mode.

The lidar has been used to evaluate the use of a point measurement of the boundary layer structure on the slant path transmission as could be derived from a NOVAM calculated extinction profile. The slant path transmission is important for use in a TDA to determine the effective range at which a certain target can be detected by some IR system.

To this end, the lidar-measured backscatter profile, which is usually assumed to be related to the extinction profile by a simple proportionality factor, has been integrated over the boundary layer. The relative difference with respect to a reference profile at an arbitrary chosen start time, was calculated over periods of up to 10 hours. Results have been presented which show that the choice of the start time strongly influences the results. When the reference profile is taken near a cloud, or in the region of cloud influence, the slant path transmission calculated from NOVAM with this sounding will be too high. This applies both short after the sounding and obviously also longer there after. The question is whether this can be avoided. In the example presented in section 5, a new sounding was taken after 5 hours. This reduced the error, but due to changes in the boundary layer resulting in a decrease in the backscatter coefficient, the error in the integrated profile rapidly increased to $50 \%$ and then fluctuated between $10 \%$ and $50 \%$. These fluctuations cannot solely be ascribed to fluctuations in the boundary layer height alone, and other effects may have played a role as well.

The question is whether these results can be used to give an error estimate for the validity of the calculated slant path transmission. At this stage the answer cannot be given. Frequency of occurrence histograms of the errors $\epsilon$ have been made for some periods. These show that on the average the errors may be between $10 \%$ and $30 \%$, although in some cases the histograms peak at much larger errors. The statistical analysis must be continued for a general assessment of the error induced by the use of a single sounding.

In addition, the convective situation, and in particular the presence of cumulus clouds, during the KEY90 experiment caused very strong variations in the boundary layer structure. These are not typical for other environments and usually the situation is somewhat simpler. Hence the error estimate will depend on the meteorological situation. To quantify the errors for the various types of situations, similar analyses must be made of data obtained during other experiments in other areas such as the North Atlantic, ${ }^{4}$ or the North Sea. ${ }^{27}$ These may yield results which are of more operational interest than those obtained during KEY90 which was characterized by fair weather and good visibilities. 
In conclusion, the KEY90 experiments have yielded a wealth of information which contributes to the further improvement of NAM and NOVAM and to the development of a generally applicable model for the extinction in the marine atmospheric boundary layer.

\section{ACKNOWLEDGEMENTS}

The investigations reported in this contribution were supported by ONR, Grants N00014-87-J-1212 and N00014-91-J-1948. The KEY90 experiments were organized in the framework of the USNAVY effort to develop a model for predicting slant path extinction in the marine atmospheric boundary layer, i.e. OSNavy (ONT 214 / NOSC 54), commonly designated the EOMET project. Dr. Stu Gathman of NRL successfully coordinated and organized the experiments. The aerosol measurements were performed by Marcel Moerman, Bert Neger assisted in the data analysis.

\section{REFERENCES}

1. S.G. Gathman, "Optical properties of the marine aerosol as predicted by the Navy aerosol model", Opt. Eng. 22, 57$62,1983$.

2. S.G. Gathman, "A preliminary description of NOVAM, the Navy Oceanic Vertical Aerosol Model", Naval Research Laboratory, Washington D.C., U.S.A., NRL Report 9200, 1989.

3. F.X. Kneizys, E.P. Shettle, w.0. Gallery, J.H. Chetwynd Jr., J.H. Abreu, J.E.A. Selby, S.A. Clough and R.W. Fenn, "Atmospheric transmittance/radiance: computer code LOWTRAN 6", AFGL-TR-83-0187, Air Force Geophysics Laboratory, Hanscom Air Force Base, Bedford, MA, USA, 1983.

4. G. de Leeuw, "Modeling of extinction and backscatter profiles in the marine mixed-layer", Appl. Opt. 28, 1356-1359, 1989.

5. G. de Leeuw,K.L. Davidson, S.G. Gathman and R.V. Noonkester, "Modeling of aerosols in the marine mixed-layer", Propagation Engineering, SPIE Proc. Vol. 1115, 27-1 to 27-8, 1989.

6. G. de Leeuw, K.L. Davidson, S.G. Gathman and R.V. Noonkester, "Physical models for aerosols in the marine mixedlayer", operational decision aids for exploiting or mitigating electromagnetic propagation effects, AGARD-CP 453 , pp. $40-1$ to $40-8,1989$.

7. K.L. Davidson, G. de Leeuw, S.G. Gathman and D. Jensen, "Verification of the Naval Oceanic Vertical Aerosol Model during FIRE," FIRE Science Results 1989, NASA Conference Publ. 3079, 191-196, 1990.

8. S.G. Gathman, G. de Leeuw, K.L. Davidson and D. Jensen, "The Naval Oceanic Vertical Aerosol Hodel: Progress Report". Atmospheric propagation in the UV, visible, IR and mm-wave region and related systems aspects, AGARDCP 454, pp. 17-1 to 17-11, 1990.

9. C.W. Fairall and K.L. Davidson, "Dynamics and modeling of aerosols in the marine atmospheric boundary layer", Oceanic Whitecaps, E.C. Monahan and G. Mac Niocaill (eds.), 195-208, D. Reidel, Dordrecht, 1986.

10. K.L. Davidson and C.W. Fairall, "Optical properties of the marine atmospheric boundary layer: aerosol profiles", Ocean Optics VIII, Proc. SPIE, Vol. 637, 18-24, 1986.

11. S.G. Gathman, "Model for estimating meteorological profiles from shipboard observations", Naval Research Laboratory, Washington D.C., O.S.A., NRL Report 8279, 1978.

12. V.R. Noonkester, "Profiles of optical extinction coefficients calculated from droplet spectra observed in marine stratus cloud layers", J. Atmos. Sci. 42, 1161-1171, 1985.

13. H.G. Hughes, "An evaluation of the LOWTRAN 6 Navy maritime aerosol model using 8- to 12- $\mu$ m sky radiances", Opt. Eng. 26, 1155-1160, 1987.

14. S.G. Gathman (1990). The KEY90 experiment plan; private communication.

15. $G$, de Leeuw, "Vertical profiles of giant particles close above the sea surface", Tellus 38B, 51-61, 1986.

16. G. de Leeuw, "Profiling of aerosol concentrations, particle size distributions and relative humidity in the atmospheric surface layer over the North Sea", Tellus 42B, 342-354, 1990.

17. G.J. Kunz, "Results from the VISA project (Vertical Infrared Structure of the Atmosphere", TNO Physics and Electronics Laboratory, report. In preparation.

18. G. de Leeuw and K.L. Davidson, "Aerosol modeling in the marine atmospheric boundary layer", Man and his ecosystem, L.J. Brasser and W.C. Mulder (Eds.), Vol. 3, 617-622, Elsevier Science Publ. B.V. Amsterdam, 1989.

19. G. de Leeuw, G.J. Kunz and M.M. Moerman, "Lidar and aerosol measurements by the Physics and Electronics Laboratory TNO during KEY90 (Marathon, Florida, USA: July 2-19, 1990)", TNO Physics and Electronics Laboratory, report FEL-90-B375, 1990 . 
20. S.G. Gathman, D.R. Jensen, W.A. Hooper, H.E. Gerber, K.L. Davidson, T. Cecere, H.H. Smith, I.E. Consterdine, G. de Leeuw, G.J. Kunz, M.M. Moerman and L.A. Brickman, "Initial NOVAM evaluation utilizing Key 90 electro-optic and meteorological data", Naval Ocean Systems Center, San Diego, CA, OSA, Technical Report, in preparation, 1992.

21. W.P. Hooper and H.E. Gerber, "Down looking lidar inversion constrained by ocean reflection and forward scatter of laser light", Appl. Opt. 25, 689-697, 1986.

22. G.J. Kunz, "The Netherlands results of inter lidar comparison Cardington 1983", Physics and Electronics Laboratory TNo, report IR 1984-30, 1984.

23. E.C. Monahan and I.G. O'Muircheartaigh, "Whitecaps and the passive remote sensing of the ocean surface", Int. J. Remote Sensing 7, 627-642, 1986.

24. R. Marks, "Preliminary investigations on the influence of rain on the production, concentration, and vertical distribution of sea salt aeros0l", J. Geophys. Res. 95, 22299-22304, 1990.

25. E.C. Monahan, "The ocean as a source for atmospheric particles", The role of air-sea exchange in geochemical cycling, P. Buat-Menard (Ed.), 129-163, Reidel, Dordrecht, 1986.

26. J. Wu, J.J. Murray and R.J. Lai, "Production and distributions of sea spray", J. Geophys. Res. 89, 8163-8169, 1984.

27. G. de Leeuw, "Mixed-layer profiling with lidar and modeling of the aerosol vertical structure", Humidity Exchange Over the Sea Main Experiment (HEXMAX) Analysis and Interpretation, W.A. Oost, S.D. Smith and K.B. Katsaros (Eds.), 100-104, Technical Report, Dept. Atmos. Sci., AK-40, Univ. Washington, Seattle, OSA, 1988.

28. W.A. Hooper and E.W. Eloranta, "Lidar measurement of wind in the planetary boundary layer: the method, accuracy and results from joint measurements with radiosonde and kytoon", J. Clim. Appl. Meteor. 25, 990-1001, 1986.

29. G.J. Kunz and G. de Leeuw, "On inversion of lidar signals with the slope method", submitted for publication.

30. K.L. Davidson, J. DeCosmo, G. de Leeuw, J.B. Edson, K.B. Katsaros, H.T. Mengelkamp, W.A. Oost, S.D. Smith and P.K. Taylor, "Variation of wind, stress, heat and vapor fluxes in vicinity of fronts", in preparation.

31. R.A. Blake, "The dependence of wind stress on wave height and wind speed", J. Geophys. Res. 96, 20531-20545, 1991.

32. N. Maat, C. Kraan and W.A. Oost, "The roughness of wind waves", Bound.-Layer Meteor. 54, 89-103, 1991. 